\title{
ATRIBUTOS QUÍMICOS DE UM LATOSSOLO BRUNO SOB SISTEMA PLANTIO DIRETO EM FUNÇÃO DA ESTRATÉGIA DE ADUBAÇÃo E DO MÉTODO DE AMOSTRAGEM DE SOLO(1)
}

\author{
Volnei Pauletti ${ }^{(2)}$, Antonio Carlos Vargas Motta ${ }^{(2)}$, Beatriz Monte Serrat ${ }^{(2)}$, Nerilde \\ Favaretto $^{(2)}$ \& Adilson dos Anjos ${ }^{(3)}$
}

\begin{abstract}
RESUMO
Adubo em sulco de semeadura nem sempre aumenta a produtividade de culturas anuais, contudo aumenta a variabilidade química das áreas agrícolas, especialmente dos nutrientes $\mathrm{P}$ e $\mathrm{K}$. Essa variabilidade é maior no sistema plantio direto por não haver homogeneização da camada superficial pelo preparo mecânico. Neste trabalho, avaliou-se, em um Latossolo Bruno, por longo período sob plantio direto, o efeito de estratégias de aplicação de adubo sobre a distribuição de alguns atributos químicos do solo em profundidade após três e seis anos do início do experimento e sobre a variação desses atributos em função de dois métodos de coleta de amostras de solo, com e sem inclusão da linha de semeadura. Foram feitos dez tratamentos, nove contemplando fontes de $P$ (fosfato natural e superfosfato triplo - STP), mecanismos sulcadores (disco duplo e haste), formas (sulco e superfície) e épocas (inverno e verão) de aplicação de adubo de semeadura e um tratamento controle, sem adubação. Os atributos químicos do solo foram maiores nas camadas superficiais e sua distribuição em profundidade, com exceção do $P$, não foi alterada pelas estratégias de adubação. $O$ teor de $P$ foi menor na profundidade de 0-5 cm com o uso contínuo, por mais de três anos, do sulcador tipo haste e com a ausência de adubação. Com a inclusão da linha de semeadura na amostragem, foram observados maiores teores de $\mathrm{K} \mathrm{e} \mathrm{Ca} \mathrm{e} \mathrm{maiores} \mathrm{valores} \mathrm{de} \mathrm{CTC.}$ Os métodos de coleta de amostras de solo, com e sem inclusão da linha de
\end{abstract}

\footnotetext{
(1) Parte da Tese de Doutorado do primeiro autor apresentada ao Programa de Pós-Graduação em Agronomia, área de concentração Produção Vegetal, Departamento de Fitotecnia e Fitossanitarismo, Setor de Ciências Agrárias, Universidade Federal do Paraná - UFPR. Recebido para publicação em julho de 2007 e aprovado em março de 2009.

(2) Professor do Departamento de Solos, Universidade Federal do Paraná - UFPR. Rua dos Funcionários 1540, Juvevê, CEP 80035050 Curitiba (PR). E-mail: vpauletti@ufpr.br, mottaacv@ufpr.br, bmserrat@ufpr.br, nfavaretto@ufpr.br.

${ }^{(3)}$ Professor do Departamento de Estatística, UFPR. E-mail: aanjos@est.ufpr.br
} 
semeadura, e as profundidades de coleta 0-10 e 0-20 cm foram semelhantes quanto à interpretação dos resultados das análises para recomendação de adubação e calagem para áreas sob sistema plantio direto, por longo período.

Termos de indexação: formas de aplicação, fertilizante, adubo, adubo a lanço.

\title{
SUMMARY: CHEMICAL PROPERTIES OF A HAPLOHUMOX UNDER NO- TILLAGE AS RELATED TO FERTILIZATION STRATEGIES AND SOIL SAMPLING METHOD
}

\begin{abstract}
Fertilizer application in the sowing rows does not necessarily increase crop yields, but it does increase the variability of the soil chemical properties, particularly of the nutrients $P$ and $K$. This variability is greater in the no-tillage system because there is no homogenization of the soil surface layer. This study evaluated the effect of fertilization strategies on soil chemical properties at distinct soil depths after three and six years from the beginning of the experiment in a Haplohumox under long-term no-tillage. Besides, the variation of these properties considering two methods of soil sampling, with and without inclusion of the seeding row, was evaluated. Ten treatments were applied, nine considering $P$ sources (rock phosphate and triple superphosphate), row preparation equipment (double disc opener and coulter opener), fertilizer placement (in-row and broadcast), time of application (summer and winter) and one control treatment, without fertilizer. The measured soil chemical properties were higher in the surface layers and depth distribution, except for $P$, was not influenced by the fertilization strategies. The soil P values were the lowest in the 0-5 cm layer where the coulter opener equipment had been continuously used for more than three years and no fertilizer was applied. With the inclusion of the sowing row in the soil sampling, higher K, Ca and CEC values were observed. The methodologies of soil sampling, with and without the inclusion of the sowing row and the $0-10$ and 0-20 $\mathrm{cm}$ sampling layers resulted in similar fertilizer and lime recommendations for areas under long-term no-tillage.
\end{abstract}

Index terms: fertilizer application forms, fertilizer, soil sampling, broadcast fertilization.

\section{INTRODUÇÃO}

A aplicação de adubos contendo N, P e K tem por objetivo atender parte ou toda a demanda dos nutrientes das culturas anuais como soja, milho e feijão. Esta aplicação geralmente é realizada com semeadoras que podem utilizar diferentes mecanismos sulcadores, depositando o adubo de forma concentrada abaixo das sementes. Entre os mecanismos mais utilizados, estão o tipo disco duplo, que abre um sulco de aproximadamente $7 \mathrm{~cm}$ de profundidade para deposição do adubo, e o tipo haste ou facão, que produz um sulco de até $15 \mathrm{~cm}$ de profundidade (Coelho, 1998).

A aplicação dos adubos em sulcos, sem posterior revolvimento do solo, aumenta as variabilidades horizontal e vertical dos atributos químicos do solo, que são intensificadas pela concentração de nutrientes e matéria orgânica nas camadas superficiais, observação unânime em áreas sob plantio direto (Muzilli, 1983; Eckert \& Johnson, 1985; Eltz et al., 1989; Merten \& Mielniczuk, 1991; Holanda et al., 1998; Howard et al., 2002; Pauletti et al., 2005). Devido a essa concentração superficial, em algumas regiões do Brasil é sugerida a coleta de amostras de solo para avaliação da fertilidade na profundidade de $0-5$ e $5-10 \mathrm{~cm}$ (CFSEMG, 1999) ou na profundidade de $0-10 \mathrm{~cm}$ (CQFS-RS/SC, 2004), substituindo a coleta na profundidade de $0-20 \mathrm{~cm}$, usualmente recomendada para sistemas que apresentam uniformização da camada superficial pelo revolvimento mecânico. No entanto, alguns autores observaram que o método de coleta para áreas com revolvimento do solo poderia ser utilizada para o plantio direto (Bordoli \& Mallarino, 1998; Stecker \& Brown, 2001).

A variabilidade horizontal é observada principalmente para $\mathrm{P} \mathrm{e} \mathrm{K}$, sendo menores para $\mathrm{pH}$ e matéria orgânica (MO) (Souza et al., 1998; Silveira et al., 2000; Schlindwein \& Anghinoni, 2000; Alvarez V. \& Guarçoni, 2003). Essa variabilidade parece não estar relacionada com os teores do nutriente no solo e tempo de cultivo sob plantio direto (Schlindwein \& Anghinoni, 2000), mas com a forma de aplicação dos adubos, especialmente a realizada em sulcos de semeadura (Kitchen et al., 1990; Klepker \& Anghinoni, 1995; Stecker \& Brown, 2001). Os efeitos residuais dessa aplicação localizada são dependentes da dose de nutriente utilizada e do tipo de solo (Stecker \& Brown, 2001). No caso do K, a concentração na linha de 
semeadura também pode ocorrer pela deposição proveniente da lavagem da parte aérea das plantas (Klepker \& Anghinoni, 1995). Portanto, a forma de aplicação do adubo pode influenciar o resultado da análise de solo para recomendação de adubação, e a participação da porção de solo adubado com P e K na amostra tende a aumentar o teor desses nutrientes na análise (Vasconcellos et al., 1982).

Diversos estudos foram realizados para definir a melhor forma de amostragem de solo no sistema plantio direto. Kitchen et al. (1990) sugeriram, quando for conhecida a localização da linha de semeadura, coletar uma amostra na linha onde foi depositado o adubo para cada 20 amostras na entrelinha, em espaçamento de semeadura de $76 \mathrm{~cm}$, ou para cada oito amostras na entrelinha em espaçamentos de $30 \mathrm{~cm}$, para compor uma amostra composta. A CQFSRS/SC, (2004) recomenda, em áreas com adubação a lanço, a coleta de 15 a 20 pontos de $3-5 \mathrm{~cm}$ de espessura, com largura de 7-10 cm. Em áreas com adubação em linha, faz-se a coleta de aproximadamente 15 pontos de $3-5 \mathrm{~cm}$ de espessura pela largura do espaçamento de semeadura da cultura anterior à coleta, usando pá-de-corte, mantendo-se a linha em que foi aplicado o adubo na parte mediana da cova. Alternativa a esta forma de coleta foi apresentada por Nicolodi et al. (2002) e CQFS-RS/SC (2004), substituindo a pá de corte por trado do tipo calador, obtendo vantagem em diminuir a quantidade de solo manipulada.

Dos trabalhos que avaliaram métodos de coleta de amostras de solo em plantio direto em função de formas de aplicação dos nutrientes, poucos verificaram a evolução dos atributos químicos, ou restringem-se a um ou dois anos de experimento.

Este trabalho teve por objetivo avaliar, em uma área cultivada por longo período sob sistema plantio direto, o efeito de estratégias de aplicação de adubo sobre a distribuição de alguns atributos químicos do solo em profundidade após três e seis anos do início do experimento e sobre a variação destes atributos químicos em função de dois métodos, com e sem inclusão da linha de semeadura, e duas profundidades, 0-10 e 0-20 cm, de coleta de amostras de solo.

\section{MATERIAL E MÉTODOS}

O experimento foi desenvolvido no Campo Demonstrativo e Experimental (CDE) da Fundação ABC em Castro, $\mathrm{PR}$, primeiro planalto paranaense, latitude $24^{\circ} 51^{\prime} 44,35$ ” S, longitude $49^{\circ} 56^{\prime} 0,40$ ” W e altitude de $1.030 \mathrm{~m}$, sul do Brasil. Pela classificação de Köppen, o clima da região é do tipo Cfb (IAPAR, 2005).

O solo é caracterizado como Latossolo Bruno distrófico típico horizonte A proeminente textura argilosa fase campo subtropical com relevo suave ondulado (Embrapa/Fundação ABC, 2001). Foram coletadas amostras de solo antes da implantação do experimento e analisadas segundo Raij et al. (1987). A área apresentou, no início do experimento, teor alto de $\mathrm{P}$ e de saturação por bases ( $\mathrm{V} \%$ ), teor médio de $\mathrm{K}$, considerando a camada de $0-20 \mathrm{~cm}$ (Quadro 1), valores maiores nas camadas superficiais, e estava sob plantio direto há mais de 10 anos, com visível variação vertical dos atributos químicos.

O ensaio foi realizado de 1998 a 2004, em uma área total de $50 \times 50 \mathrm{~m}$. A rotação de culturas adotada foi aveia-preta/ soja/ aveia-preta/ milho/ trigo/ feijão. Os cultivares utilizados foram: comum, BRS 133, XL 214, CD 105 e carioca comum, de aveia-preta, soja, milho, trigo e feijão, respectivamente. O espaçamento entre as linhas foi de $80 \mathrm{~cm}$ para o milho e $40 \mathrm{~cm}$ para a soja e o feijão.

O delineamento experimental foi de blocos ao acaso com quatro blocos e 10 tratamentos. O tamanho de cada unidade experimental (parcela) foi de $5,55 \times 12,5 \mathrm{~m}$, sendo considerada área útil $3,2 \times 8,5 \mathrm{~m}$, ou seja, $27,2 \mathrm{~m}^{2}$, onde todas as avaliações foram realizadas.

Os tratamentos avaliados (Quadro 2) combinaram fontes de $\mathrm{P}$, mecanismos sulcadores e formas e épocas de aplicação do adubo de semeadura, sendo aplicados $\mathrm{N}, \mathrm{P}$ e K para milho e feijão e somente $\mathrm{P}$ e K para a soja. Foram aplicados para milho 30,26 e $50 \mathrm{~kg} \mathrm{ha}^{-1}$, para feijão 30,26 e $33 \mathrm{~kg} \mathrm{ha}^{-1}$ e para soja 0,26 e $33 \mathrm{~kg} \mathrm{ha}^{-1}$ de $\mathrm{N}, \mathrm{P}$ e K, respectivamente. No milho, junto com esses nutrientes, foram aplicados $3 \mathrm{~kg} \mathrm{ha}^{-1}$

Quadro 1. Caracterização química e textural da área, antes do início do experimento (1998)

\begin{tabular}{|c|c|c|c|c|c|c|c|c|c|c|c|}
\hline Profundidade & $\mathbf{p} \mathbf{H}^{(1)}$ & $\mathbf{P}^{(2)}$ & $\mathrm{CO}^{(3)}$ & $\mathbf{K}$ & $\mathbf{C a}$ & Mg & $\mathbf{V}^{(4)}$ & Al & Argila $^{(5)}$ & Areia $^{(5)}$ & Silte ${ }^{(5)}$ \\
\hline $\mathrm{cm}$ & & $\mathrm{mg} \mathrm{dm}^{-3}$ & $\mathrm{~g} \mathrm{dm}^{-3}$ & \multicolumn{3}{|c|}{$-\mathrm{mmol}_{\mathrm{c}} \mathrm{dm}^{-3}-$} & \multicolumn{2}{|c|}{$-\%$} & - & $-\mathrm{g} \mathrm{kg}^{-1}$ & 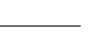 \\
\hline $0-5$ & 5,9 & 122 & 38 & 4,0 & 66 & 39 & 78 & 0 & 607 & 136 & 257 \\
\hline $5-10$ & 5,4 & 43 & 34 & 2,6 & 56 & 32 & 64 & 0 & 622 & 136 & 242 \\
\hline $10-30$ & 5,1 & 11 & 28 & 1,4 & 34 & 16 & 47 & 1,9 & 627 & 150 & 223 \\
\hline $0-20$ & 5,2 & 51 & 30 & 2,2 & 43 & 30 & 60 & 0 & 616 & 131 & 253 \\
\hline
\end{tabular}

${ }^{(1)} \mathrm{pH}_{\text {em } \mathrm{CaCl}_{2} \cdot{ }^{(2)} \text { Resina aniônica. }}{ }^{(3)}$ Carbono orgânico. ${ }^{(4)}$ Saturação por bases. ${ }^{(5)}$ Método da pipeta. 
de Zn. Com exceção das parcelas do tratamento 10 , foram aplicados no estádio V3/V4 no milho e no feijão, a lanço e sem incorporação, 90 e $35 \mathrm{~kg} \mathrm{ha}^{-1}$ de N, respectivamente. Como fonte desses nutrientes, utilizaram-se a uréia, o $\mathrm{KCl}$ e o superfosfato triplo (SFT), com exceção do tratamento 4, em que foi aplicado fosfato natural em substituição ao SFT, e do tratamento 10 , sem adubação. O fosfato natural utilizado foi o Carolina do Norte até a safra 2001/2002 e, após esta safra, o fosfato de Gafsa, ambos na forma farelada, sendo considerado o teor total de $\mathrm{P}$ para o cálculo da quantidade a ser aplicada. Imediatamente antes da aplicação, procedeu-se à mistura dessas fontes para a obtenção das quantidades de nutrientes necessárias. Os mecanismos sulcadores foram o do tipo haste da empresa Semeato, também conhecido como guilhotina ${ }^{\circledR}$ (Semeato, 2006), com medidas de $2,5 \mathrm{~cm}$ na ponteira, $4,0 \mathrm{~cm}$ na maior largura da haste e $11 \mathrm{~cm}$ de profundidade de trabalho e o do tipo disco duplo defasado composto por dois discos, sendo um de $339 \mathrm{~mm}$ (14") e outro de $363 \mathrm{~mm}$ (15"), com aproximadamente $8 \mathrm{~cm}$ de profundidade de trabalho. Os dois mecanismos sulcadores trabalharam alinhados ao sulco de deposição das sementes. No primeiro, o adubo foi colocado a aproximadamente 5$6 \mathrm{~cm}$ abaixo da semente, enquanto no segundo (disco duplo) o adubo foi depositado cerca de $2-3 \mathrm{~cm}$ abaixo das sementes.

Quanto à adubação das culturas de inverno, foram aplicados no trigo $110 \mathrm{~kg} \mathrm{ha}^{-1}$ de $\mathrm{N}$ e doses de $\mathrm{P}$ e K para reposição desses nutrientes exportados pela colheita, seguindo-se os dados apresentados por Pauletti (1998). A aveia-preta não foi adubada. A aplicação dos adubos no inverno foi realizada manualmente a lanço antes da semeadura em todas as parcelas, somando-se à aplicação dos nutrientes da cultura de inverno (no caso do trigo) e de verão para os tratamentos 1, 2, 3 e 4 (Quadro 2).

Para avaliar a distribuição de alguns atributos da fertilidade do solo em profundidade, foram coletadas amostras de solo nas profundidades de 0-5, 5-10 e 10-20 cm em 2001 e 2004, após as colheitas de verão, em todas as parcelas. Essas datas coincidem com a conclusão de um e dois ciclos da rotação, respectivamente, ou seja, três e seis anos após o início do experimento. Na amostragem de 2001, coletaramse 20 pontos por parcela para formar uma amostra composta, utilizando-se trado tipo calador nas entrelinhas do feijão colhido. Na amostragem de 2004, adotou-se procedimento segundo Nicolodi et al. (2002) e CQFS-RS/SC, (2004), coletando-se, com trado tipo calador, sete amostras transversais ao sentido da semeadura, sendo uma na linha de semeadura da cultura anterior e três em cada lado, em oito locais por parcela, totalizando 56 amostras simples por parcela. Para simplificar citações posteriores, foi denominado entrelinha o procedimento adotado em 2001 e linha o adotado em 2004.

Para comparar os métodos de amostragem em 2004, seis anos após o início do experimento, foram coletadas amostras de solo nas profundidades $0-10 \mathrm{e}$ $0-20 \mathrm{~cm}$ tanto no procedimento linha como no entrelinha em todas as parcelas.

As determinações químicas foram feitas no Laboratório da Fundação ABC em Castro-PR, de acordo com Raij et al. (1987). A extração de P, Ca, $\mathrm{Mg}$ e $\mathrm{K}$ foi feita com resinas trocadoras de íons, sendo a determinação do $\mathrm{P}$ por colorimetria e dos demais por espectrofotometria de absorção atômica. $\mathrm{O}$ pH foi

\section{Quadro 2. Época e forma de aplicação dos nutrientes e mecanismo sulcador utilizado em cada cultura, por tratamento}

\begin{tabular}{|c|c|c|c|c|c|c|}
\hline \multirow{3}{*}{ Tratamento } & \multicolumn{3}{|c|}{ Época e forma de aplicação do nutriente ${ }^{(1)}$} & & & \\
\hline & \multirow{2}{*}{$\begin{array}{c}\text { Inverno }^{(2)} \\
\text { Lanço }\end{array}$} & \multicolumn{2}{|c|}{ Verão } & \multicolumn{3}{|c|}{ Mecanismo sulcador por cultura } \\
\hline & & Sulco & $\operatorname{Lanço}^{(3)}$ & Soja & Milho & Feijão \\
\hline 1 & NPK & - & - & Disco duplo & Haste & Haste \\
\hline 2 & $\mathrm{P}$ & NK & - & Disco duplo & Haste & Haste \\
\hline 3 & $\mathrm{P}$ & - & NK & Disco duplo & Haste & Haste \\
\hline 4 & $\mathrm{P}-\mathrm{FN}^{(4)}$ & NK & - & Disco duplo & Haste & Haste \\
\hline 5 & - & NPK & - & Disco duplo & Disco duplo & Disco duplo \\
\hline 6 & - & NPK & - & Haste & Haste & Haste \\
\hline 7 & - & $\mathrm{NPK}^{(5)}$ & - & Disco duplo & Haste & Haste \\
\hline 8 & - & - & NPK & Disco duplo & Haste & Haste \\
\hline 9 & - & NP & $\mathrm{K}$ & Disco duplo & Haste & Haste \\
\hline 10 & - & - & - & Disco duplo & Haste & Haste \\
\hline
\end{tabular}

${ }^{(1)} \mathrm{Na}$ soja não foi aplicado N. ${ }^{(2)}$ Em toda a área, imediatamente antes da semeadura, sem incorporação. ${ }^{(3)}$ Em toda a área, imediatamente após a semeadura. ${ }^{(4)}$ Fosfato natural Carolina do Norte até a safra 2001/2002 e, posteriormente, Gafsa. ${ }^{(5)}$ Aplicado em faixa sobre a linha de semeadura. 
determinado em $\mathrm{CaCl}_{2}$,, $01 \mathrm{~mol} \mathrm{~L}^{-1}$ e a acidez potencial $(\mathrm{H}+\mathrm{Al})$ por solução tampão SMP. O carbono orgânico (CO) foi determinado pelo método WalkleyBlack modificado.

Antes da análise estatística foram eliminados os dados discrepantes (outliers), identificados por meio da distribuição normal padrão, utilizando o critério de três desvios-padrão para mais ou para menos. Para avaliar os efeitos dos tratamentos na distribuição dos componentes da fertilidade do solo em profundidade, procedeu-se à análise de variância individual por ano, utilizando-se o delineamento em blocos completos ao acaso, em esquema de parcela subdividida, sendo a parcela o tratamento e a subparcela a profundidade de coleta. Para comparar os métodos de amostragem de solo em função dos tratamentos e das profundidades, utilizando os dados obtidos em 2004, realizou-se a análise de variância com o delineamento blocos ao acaso em esquema fatorial $10 \times 2 \times 2$, comparando-se 10 tratamentos (Quadro 2), duas profundidades $(0-10$ e $0-20 \mathrm{~cm})$ e dois métodos de coleta (linha e entrelinha). Adotou-se o nível de significância de $5 \%$. Em caso de análise de variância significativa, aplicou-se o teste de Tukey para comparação das médias (Montgomery, 2001). As análises estatísticas foram realizadas no programa R 2.1.1 (R Development, 2005).

\section{RESULTADOS E DISCUSSÃO}

\section{Distribuição de atributos da fertilidade do solo em profundidade - três anos após o início do experimento}

Não houve interação entre profundidade e os tratamentos com relação às variáveis. Os teores foram maiores, quanto mais superficial foi a amostra (Quadro 3). Esta concentração superficial foi observada por diversos autores (Muzilli, 1983; Eckert \& Johnson, 1985; Eltz et al., 1989; Merten \& Mielniczuk, 1991; Holanda et al., 1998; Howard et al., 2002; Pauletti et al., 2005) e ocorre pela falta de revolvimento do solo. No caso do $\mathrm{P}$, também pode ocorrer no plantio direto menor adsorção na camada superficial devido ao recobrimento dos sítios de adsorção por radicais orgânicos e substituição de íons fosfatos por íons orgânicos nesses sítios (Tisdale et al., 1985), além do menor contato desse elemento com os sítios de adsorção por falta de revolvimento.

Os teores de $\mathrm{P}$ foram considerados altos em todas as profundidades e os de $\mathrm{K}$ altos até $10 \mathrm{~cm}$ e médios na camada de 10-20 cm (Quadro 3). Entre os tratamentos, não houve diferença nos teores de $\mathrm{P}, \mathrm{K}$, $\mathrm{CO}$, Ca e Mg, e valores de CTC e V \% no solo. Em

Quadro 3. Teores de fósforo, carbono orgânico, potássio, cálcio e magnésio, e valores de pH, capacidade de troca de cátions e saturação por bases no solo, em função dos tratamentos e da profundidade das amostras - coletadas nas entrelinhas de semeadura - 2001

\begin{tabular}{|c|c|c|c|c|c|c|c|c|}
\hline Tratamento $^{(1)}$ & $\mathbf{P}$ & $\mathrm{CO}$ & $\mathbf{p} \mathbf{H}_{\mathrm{CaCl}_{2}}$ & $\mathbf{K}$ & $\mathrm{Ca}^{2+}$ & $\mathbf{M g}^{2+}$ & CTC & V \\
\hline & $\mathrm{mg} \mathrm{dm}^{-3}$ & $\mathrm{~g} \mathrm{dm}^{-3}$ & & \multicolumn{4}{|c|}{$-\mathrm{mmol}_{\mathrm{c}} \mathrm{dm}^{-3}$} & $\%$ \\
\hline $1^{(2)}$ & 74 & 32 & 5,0 & 3,5 & 55 & 23 & 141 & 57 \\
\hline $2^{(2)}$ & 67 & 31 & 5,1 & 2,8 & 57 & 24 & 141 & 59 \\
\hline $3^{(2)}$ & 85 & 32 & 5,2 & 2,5 & 66 & 27 & 148 & 64 \\
\hline $4^{(2)}$ & 67 & 32 & 5,0 & 2,8 & 54 & 25 & 141 & 56 \\
\hline 5 & 83 & 32 & 5,2 & 2,6 & 62 & 27 & 144 & 62 \\
\hline 6 & 77 & 33 & 5,1 & 2,8 & 58 & 25 & 144 & 59 \\
\hline $7^{(2)}$ & 66 & 32 & 5,1 & 2,3 & 57 & 24 & 140 & 59 \\
\hline $8^{(2)}$ & 70 & 33 & 5,1 & 3,0 & 56 & 26 & 141 & 60 \\
\hline $9^{(2)}$ & 86 & 33 & 5,2 & 3,1 & 62 & 27 & 141 & 65 \\
\hline $10^{(2)}$ & 60 & 34 & 5,1 & 2,4 & 54 & 25 & 143 & 57 \\
\hline Prob > F & 0,217 & 0,313 & 0,299 & 0,082 & 0,109 & 0,363 & 0,623 & 0,440 \\
\hline \multicolumn{9}{|c|}{ Profundidade $(\mathrm{cm})$} \\
\hline $0-5$ & $131 \mathrm{a}^{(3)}$ & $38 \mathrm{a}$ & $5,3 \mathrm{a}$ & $3,9 \mathrm{a}$ & $76 \mathrm{a}$ & $33 \mathrm{a}$ & $158 \mathrm{a}$ & $71 \mathrm{a}$ \\
\hline $5-10$ & $90 \mathrm{~B}$ & $31 \mathrm{~b}$ & $5,1 \mathrm{~b}$ & $3,2 \mathrm{~b}$ & $56 \mathrm{~b}$ & $24 \mathrm{~b}$ & $142 \mathrm{~b}$ & $59 \mathrm{~b}$ \\
\hline $10-20$ & $57 \mathrm{C}$ & $28 \mathrm{c}$ & $4,9 \mathrm{c}$ & $2,6 \mathrm{c}$ & $42 \mathrm{c}$ & $19 \mathrm{c}$ & $128 \mathrm{c}$ & $49 \mathrm{c}$ \\
\hline
\end{tabular}

(1) Aplicação de N, P, K: 1 = a lanço em toda a área, imediatamente antes da semeadura da cultura de inverno, sem incorporação; $2=\mathrm{P}$ como em 1 e N e K no sulco de semeadura de verão; $3=\mathrm{P}$ como em 1 e N e K a lanço em toda a área, imediatamente antes da semeadura de verão; 4 = idem ao 2 , substituindo fosfato solúvel por fosfato natural Carolina do Norte até a safra 2001/2002 e posteriormente Gafsa; $5=\mathrm{N}, \mathrm{P}$ e K aplicados no sulco de semeadura de verão com sulcador do tipo disco duplo; $6=$ idem 5 , utilizando sulcador do tipo haste; $7=\mathrm{N}, \mathrm{P}$ e K aplicados em faixa sobre a linha de semeadura; $8=\mathrm{N}, \mathrm{P}$ e K aplicados a lanço em toda área, imediatamente após a semeadura de verão; $9=\mathrm{N}$ e $\mathrm{P}$ aplicados no sulco de semeadura de verão e $\mathrm{K}$ a lanço em toda a área, imediatamente após a semeadura de verão; 10 = sem aplicação de N, P e K. ${ }^{(2)}$ Utilização de mecanismo sulcador do tipo haste no feijão e no milho e do tipo disco duplo na soja. ${ }^{(3)}$ Valores seguidos da mesma letra na coluna não diferem entre si pelo teste de Tukey a $5 \%$. 
2001, a coleta foi realizada na entrelinha da cultura anterior, fato que, somado ao pouco tempo entre o início do experimento e o da coleta das amostras de solo, pode justificar a uniformidade dos resultados. Com exceção do $\mathrm{P}$ e do $\mathrm{K}$, não eram esperadas variações significativas entre os tratamentos.

Distribuição de atributos da fertilidade do solo em profundidade - seis anos após o início do experimento

Em 2004, os valores de todas as variáveis foram maiores, quanto mais superficial foi a amostra (Quadro 4), situação semelhante à observada na coleta realizada em 2001. Os teores de $\mathrm{P}$ e $\mathrm{K}$ também foram altos nas profundidades $0-5$ e $5-10 \mathrm{~cm}$, porém médios ou baixos na camada de 10-20 cm (Quadro 4). A ocorrência de teores médios ou baixos na profundidade 10-20 cm pode ser devido à não-inclusão da porção adubada, pois a aplicação do adubo nos tratamentos foi realizada em superfície ou até a profundidade aproximada de $10 \mathrm{~cm}$ com o uso da haste. Ressaltase que essa coleta foi realizada incluindo a linha de semeadura, que coincide com a de adubação.

Nas amostras coletadas em 2004, também não foram detectadas variações nos valores de $\mathrm{K}, \mathrm{CO}, \mathrm{pH}$,
Ca, $\mathrm{Mg}$, CTC e V \% com a adubação e com as diversas formas de aplicação de adubo (Quadro 4). No entanto, houve interação entre profundidade e tratamentos com relação ao $\mathrm{P}$. Na profundidade de $0-5 \mathrm{~cm}$, menores teores de $\mathrm{P}$ foram observados no tratamento 10 , com ausência de aplicação de P. No tratamento 6 , onde sempre foi utilizado o mecanismo sulcador tipo haste para incorporar o adubo, o teor de P na camada 0$5 \mathrm{~cm}$ se assemelhou ao do tratamento 10 , sendo menor que o do tratamento 5 , onde sempre foi utilizado o mecanismo sulcador disco duplo. A haste proporciona a aplicação de adubo em sulcos de até $15 \mathrm{~cm}$ (Coelho, 1998), profundidade maior que a do disco duplo, justificando este menor teor na superfície. Nas camadas de 5-10 e 10-20 cm, não houve efeito da aplicação e das formas de aplicação de adubo, situação esperada pela pouca mobilidade desse nutriente no perfil do solo. Por outro lado, a aplicação em sulco utilizando haste não aumentou o teor desse nutriente nas camadas de 5-10 e 10-20 cm, profundidade onde o adubo foi depositado. O uso de fosfato natural (tratamento 4) manteve semelhança aos tratamentos com uso de fosfato solúvel, demonstrando que as duas fontes de $\mathrm{P}$ apresentam a mesma capacidade de manutenção em longo prazo do P disponível no solo avaliado pela metodologia da resina.

Quadro 4. Teores de fósforo, carbono orgânico, potássio, cálcio e magnésio, e valores de pH, capacidade de troca de cátions (CTC) e saturação por bases (V) no solo, em função dos tratamentos e da profundidade das amostras - coletadas incluindo a linha de semeadura - 2004

\begin{tabular}{|c|c|c|c|c|c|c|c|c|c|c|}
\hline \multirow[t]{2}{*}{ Tratamento $^{(1)}$} & \multicolumn{3}{|c|}{$\mathbf{P}$} & $\mathrm{CO}$ & $\mathbf{p} \mathbf{H}_{\mathrm{CaCl}_{2}}$ & $\mathbf{K}$ & $\mathrm{Ca}^{2+}$ & $\mathbf{M g}^{2+}$ & CTC & $\mathbf{V}$ \\
\hline & \multicolumn{3}{|c|}{$\mathrm{mg} \mathrm{dm}^{-3}$} & \multirow[t]{3}{*}{$\mathrm{g} \mathrm{dm}-3$} & & & \multirow{3}{*}{\multicolumn{2}{|c|}{$\mathrm{mmol}_{\mathrm{c}} \mathrm{dm}^{-3}$}} & & \multirow[t]{3}{*}{$\%$} \\
\hline & \multicolumn{3}{|c|}{ Profundidade $(\mathrm{cm})$} & & & & & & & \\
\hline & $0-5$ & $5-10$ & $10-20$ & & & & & & & \\
\hline $1^{(2)}$ & $118 \mathrm{a}$ & 55 & 17 & 31 & 5,1 & 3,1 & 68 & 26 & 153 & 63 \\
\hline $2^{(2)}$ & $107 \mathrm{ab}$ & 50 & 16 & 31 & 5,2 & 3,0 & 66 & 26 & 147 & 64 \\
\hline $3^{(2)}$ & $118 \mathrm{a}$ & 58 & 19 & 31 & 5,3 & 2,8 & 73 & 28 & 156 & 66 \\
\hline $4^{(2)}$ & $114 \mathrm{a}$ & 46 & 11 & 31 & 5,1 & 2,6 & 65 & 26 & 144 & 64 \\
\hline 5 & 108 a & 46 & 15 & 30 & 5,3 & 2,6 & 69 & 28 & 146 & 68 \\
\hline 6 & $78 \mathrm{bc}$ & 57 & 16 & 31 & 5,1 & 2,7 & 62 & 26 & 144 & 62 \\
\hline $7^{(2)}$ & $101 \mathrm{ab}$ & 54 & 16 & 30 & 5,1 & 2,2 & 65 & 26 & 146 & 63 \\
\hline $8^{(2)}$ & $103 \mathrm{ab}$ & 42 & 14 & 31 & 5,2 & 2,7 & 69 & 28 & 149 & 66 \\
\hline $9^{(2)}$ & $91 \mathrm{abc}$ & 50 & 20 & 31 & 5,3 & 2,9 & 64 & 26 & 139 & 68 \\
\hline $10^{(2)}$ & $70 \mathrm{c}$ & 43 & 16 & 33 & 5,2 & 2,1 & 69 & 28 & 151 & 64 \\
\hline Prob $>\mathrm{F}$ & $<0,001$ & 0,298 & 0,938 & 0,220 & 0,824 & 0,068 & 0,410 & 0,757 & 0,123 & 0,747 \\
\hline \multicolumn{11}{|c|}{ Profundidade $(\mathrm{cm})$} \\
\hline $0-5$ & - & - & - & $37 \mathrm{a}^{(3)}$ & $5,5 \mathrm{a}$ & $3,9 \mathrm{a}$ & $84 \mathrm{a}$ & $33 \mathrm{a}$ & $161 \mathrm{a}$ & $75 \mathrm{a}$ \\
\hline $5-10$ & - & - & - & $30 \mathrm{~b}$ & $5,2 \mathrm{~b}$ & $2,6 \mathrm{~b}$ & $70 \mathrm{~b}$ & $27 \mathrm{~b}$ & $150 \mathrm{~b}$ & $66 \mathrm{~b}$ \\
\hline $10-20$ & - & - & - & $26 \mathrm{c}$ & $4,9 \mathrm{c}$ & $1,6 \mathrm{c}$ & $48 \mathrm{c}$ & $20 \mathrm{c}$ & $132 \mathrm{c}$ & $53 \mathrm{c}$ \\
\hline
\end{tabular}

(1) Tratamento, aplicação de N, P, K: 1 = a lanço em toda a área, imediatamente antes da semeadura da cultura de inverno, sem incorporação; $2=\mathrm{P}$ como em 1 e $\mathrm{N}$ e $\mathrm{K}$ no sulco de semeadura de verão; $3=\mathrm{P}$ como em 1 e $\mathrm{N}$ e $\mathrm{K}$ a lanço em toda a área, imediatamente antes da semeadura de verão; 4 =idem ao 2, substituindo fosfato solúvel por fosfato natural Carolina do Norte até a safra 2001/2002 e posteriormente Gafsa; $5=\mathrm{N}, \mathrm{P}$ e K aplicados no sulco de semeadura de verão com sulcador do tipo disco duplo; 6 = idem 5, utilizando sulcador do tipo haste; $7=\mathrm{N}$, P e K aplicados em faixa sobre a linha de semeadura; $8=\mathrm{N}$, P e K aplicados a lanço em toda área, imediatamente após a semeadura de verão; $9=\mathrm{N}$ e $\mathrm{P}$ aplicados no sulco de semeadura de verão e K a lanço em toda a área, imediatamente após a semeadura de verão; e; 10 = sem aplicação de $\mathrm{N}$, $\mathrm{P}$ e $\mathrm{K}$. ${ }^{(2)}$ Utilização de mecanismo sulcador do tipo haste no feijão e no milho e do tipo disco duplo na soja. ${ }^{(3)}$ Valores seguidos da mesma letra na coluna não diferem entre si pelo teste de Tukey a $5 \%$. 
Percebe-se que em condições iniciais de solo semelhantes às deste experimento, com exceção do $\mathrm{P}$, pode não ocorrer modificações na distribuição da maioria dos atributos da fertilidade do solo até seis anos após a adoção de diferentes estratégias de aplicação ou da ausência de aplicação de adubos. Para o P, no entanto, as variações ocorrem após período superior a três anos, iniciando nos centímetros superficiais.

Apesar de não ser objeto de comparação estatística neste trabalho, os valores obtidos em 2001 são maiores que os de 2004, mesmo com a inclusão da linha de semeadura, coincidente com a de adubação na coleta feita nessa última data. Também percebe-se maior concentração superficial na segunda coleta, tanto para P quanto para K. Percentualmente, a camada de 10 $20 \mathrm{~cm}$ apresentou teores de $\mathrm{P}$ e de $\mathrm{K}$ equivalentes a 44 e $67 \%$ em relação à profundidade $0-5 \mathrm{~cm}$ em 2001 , respectivamente. Os percentuais para as mesmas comparações foram de 16 e $41 \%$, respectivamente, na coleta feita em 2004. Essa constatação possivelmente reflete a deposição dos fertilizantes, ou mesmo da palha, nas camadas mais superficiais ou ainda o maior volume de solo amostrado em 2004 . Esse maior volume de solo pode ter proporcionado a diluição dos teores, situação observada com o $\mathrm{P}$ por Alvarez V. \& Guarçoni (2003), que compararam o uso de pá de corte com o trado de caneca para a coleta de amostras, encontrando menores valores com o uso da pá.

\section{Métodos de coleta de amostra}

Não houve interação entre os efeitos dos tratamentos, profundidade de coleta e método de coleta nos atributos químicos avaliados. Os tratamentos influenciaram os valores de $\mathrm{P}, \mathrm{K}, \mathrm{pH}, \mathrm{Mg}$ e CTC (Quadro 5). A variação encontrada para $\mathrm{pH}, \mathrm{Mg}$ e CTC não representa variação que possa ser considerada como efeito direto dos tratamentos. O teor de $\mathrm{P}$ foi maior nos tratamentos com aplicação deste nutriente em relação ao tratamento 10 (sem aplicação). Mesmo assim, todos os valores encontravamse em grandezas consideradas altas. O mesmo ocorreu com o $\mathrm{K}$, e a maior variação ocorreu entre a ausência de adubação (tratamento 10) e os demais tratamentos sem modificar a interpretação dos dados. Essas informações sugerem que seis anos sem adubação não foram suficientes para alterar os teores a ponto de interferir na recomendação de adubação com esses nutrientes.

Quadro 5. Teores de fósforo, carbono orgânico, potássio, cálcio e magnésio, e valores de pH, capacidade de troca de cátions (CTC) e saturação por bases (V) no solo, em função dos tratamentos, da profundidade e do método de coleta das amostras - 2004

\begin{tabular}{|c|c|c|c|c|c|c|c|c|}
\hline Tratamento $^{(1)}$ & $\mathbf{P}$ & $\mathrm{CO}$ & $\mathbf{p} \mathrm{H}_{\mathrm{CaCl}_{2}}$ & $\mathbf{K}$ & $\mathrm{Ca}^{2+}$ & $\mathrm{Mg}^{2+}$ & CTC & $\mathbf{V}$ \\
\hline & $\mathrm{mg} \mathrm{dm}^{-3}$ & $\mathrm{~g} \mathrm{dm}-3$ & & 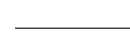 & $-\mathrm{mmol}_{\mathrm{c}}$ & $\mathrm{dm}^{-3}$ & & $\%$ \\
\hline $1^{(2)}$ & 58 a & 31 & 5,19 abc & $2,6 \mathrm{abc}$ & 65 & $25 \mathrm{~b}$ & $144 \mathrm{bc}$ & $64 \mathrm{a}$ \\
\hline $2^{(2)}$ & 55 a & 31 & $5,22 \mathrm{abc}$ & 2,9 a & 65 & $27 \mathrm{ab}$ & $145 \mathrm{bc}$ & $65 \mathrm{a}$ \\
\hline $3^{(2)}$ & $60 \mathrm{a}$ & 31 & $5,13 \mathrm{~b}$ & $2,7 \mathrm{ab}$ & 70 & $27 \mathrm{ab}$ & $154 \mathrm{~A}$ & $64 \mathrm{a}$ \\
\hline $4^{(2)}$ & 55 a & 31 & 5,19 abc & $2,2 \mathrm{bcd}$ & 66 & $26 \mathrm{ab}$ & $143 \mathrm{bc}$ & $65 \mathrm{a}$ \\
\hline 5 & $54 \mathrm{ab}$ & 31 & 5,27 a & $2,2 \mathrm{bcd}$ & 67 & $27 \mathrm{ab}$ & $143 \mathrm{bc}$ & $67 \mathrm{a}$ \\
\hline 6 & $49 \mathrm{ab}$ & 31 & 5,18 abc & $2,4 \mathrm{abc}$ & 64 & $26 \mathrm{ab}$ & $143 \mathrm{bc}$ & $64 \mathrm{a}$ \\
\hline $7^{(2)}$ & $57 \mathrm{a}$ & 31 & $5,12 \mathrm{c}$ & $2,1 \mathrm{~cd}$ & 68 & $26 \mathrm{ab}$ & $152 \mathrm{ab}$ & $63 \mathrm{a}$ \\
\hline $8^{(2}$ & $53 \mathrm{ab}$ & 32 & $5,23 \mathrm{abc}$ & $2,5 \mathrm{abc}$ & 66 & $28 \mathrm{ab}$ & $145 \mathrm{bc}$ & $66 \mathrm{a}$ \\
\hline $9^{(2)}$ & $54 \mathrm{ab}$ & 30 & $5,26 \mathrm{ab}$ & $2,7 \mathrm{ab}$ & 63 & $26 \mathrm{ab}$ & $139 \mathrm{C}$ & $66 \mathrm{a}$ \\
\hline $10^{(2)}$ & $40 \mathrm{~b}$ & 32 & 5,19 abc & $1,8 \mathrm{~d}$ & 65 & $28 \mathrm{a}$ & $147 \mathrm{abc}$ & $65 \mathrm{a}$ \\
\hline Prob $>$ F & $<0,0001$ & 0,098 & 0,002 & $<0,0001$ & 0,267 & 0,031 & $<0,0001$ & 0,042 \\
\hline \multicolumn{9}{|c|}{ Profundidade $(\mathrm{cm})$} \\
\hline $0-10$ & $66 \mathrm{a}^{(3)}$ & 33 a & 5,28 a & $2,8 \mathrm{a}$ & 71 a & $28 \mathrm{a}$ & $150 \mathrm{~A}$ & 68 a \\
\hline $0-20$ & $41 \mathrm{~b}$ & $30 \mathrm{~b}$ & $5,12 \mathrm{~b}$ & $2,1 \mathrm{~b}$ & $61 \mathrm{~b}$ & $24 \mathrm{~b}$ & $142 \mathrm{~B}$ & $62 \mathrm{~b}$ \\
\hline \multicolumn{9}{|l|}{ Coleta } \\
\hline Linha & 53 & 31 & 5,18 & 2,6 & 67 & 27 & 147 & 65 \\
\hline Entrelinha & 54 & 31 & 5,22 & 2,2 & 65 & 26 & 144 & 64 \\
\hline Prob $>$ F & 0,366 & 0,502 & 0,063 & $<0,0001$ & 0,038 & 0,162 & 0,011 & 0,145 \\
\hline
\end{tabular}

(1) Aplicação de N, P, K: 1 = a lanço em toda a área, imediatamente antes da semeadura da cultura de inverno, sem incorporação; $2=\mathrm{P}$ como em 1 e N e K no sulco de semeadura de verão; $3=\mathrm{P}$ como em 1 e N e K a lanço em toda a área, imediatamente antes da semeadura de verão; 4 = idem ao 2, substituindo fosfato solúvel por fosfato natural Carolina do Norte até a safra $2001 / 2002$ e posteriormente Gafsa; $5=\mathrm{N}, \mathrm{P}$ e K aplicados no sulco de semeadura de verão com sulcador do tipo disco duplo; 6 = idem 5 , utilizando sulcador do tipo haste; $7=\mathrm{N}, \mathrm{P}$ e K aplicados em faixa sobre a linha de semeadura; $8=\mathrm{N}$, P e K aplicados a lanço em toda área, imediatamente após a semeadura de verão; $9=\mathrm{N}$ e $\mathrm{P}$ aplicados no sulco de semeadura de verão e $\mathrm{K}$ a lanço em toda a área, imediatamente após a semeadura de verão; $10=$ sem aplicação de $\mathrm{N}, \mathrm{P}$ e K. ${ }^{(2)}$ Utilização de mecanismo sulcador do tipo haste no feijão e no milho e do tipo disco duplo na soja. ${ }^{(3)}$ Valores seguidos da mesma letra na coluna não diferem entre si pelo teste de Tukey a $5 \%$. 
Todos os atributos químicos avaliados apresentaram maior valor na coleta realizada na profundidade de $0-10 \mathrm{~cm}$ em relação à de 0-20 cm (Quadro 5). Os teores de $\mathrm{P}$ e de $\mathrm{K}$, por exemplo, foram em média $61 \mathrm{e}$ $33 \%$ maiores, respectivamente, na amostragem de 0-10 cm. Este maior teor na coleta superficial reflete a concentração superficial dos elementos químicos em função da falta de revolvimento do solo. Apesar dos maiores teores na amostragem de $0-10 \mathrm{~cm}$, não ocorreu alteração nos níveis de interpretação em relação à amostragem na profundidade de $0-20 \mathrm{~cm}$, sugerindo que, em situações (teores altos de $\mathrm{P}$ e demais elementos e teores médios de K), a profundidade de coleta não interfere na recomendação de adubação, ou mesmo de calcário. Esses dados estão de acordo com os de Bordoli \& Mallarino (1998) e Stecker \& Brown (2001), que observaram que a recomendação da profundidade de coleta de amostras sugerida para sistemas com revolvimento do solo poderia ser utilizada para o plantio direto.

A coleta de amostras que inclui a linha de adubação proporcionou maiores teores de Ca e maiores valores de CTC na análise em relação à coleta nas entrelinhas (Quadro 5). A aplicação de superfosfato triplo, que contém cerca de $10 \%$ de $\mathrm{Ca}$, no sulco pode ter sido a causa desses maiores teores, já que o Ca apresenta pouca mobilidade no solo e na planta. O maior teor de K observado na coleta que inclui o sulco de adubação (linha) deve estar relacionado à concentração no sulco pela adubação e movimentação desse nutriente com a água do solo em direção ao sistema radicular, além da lavagem da própria planta (Klepker \& Anghinoni, 1995). Apesar do maior teor de K na amostra que inclui a linha de adubação, não ocorreu mudança no nível de interpretação para recomendação desse nutriente para as culturas.

Não houve variação do teor de $\mathrm{P}$ entre os métodos avaliados: linha e entrelinha (Quadro 5). Essa constatação está em discordância com vários autores que observaram maior teor de $\mathrm{P}$ nas análises de amostras com maior participação da porção do solo adubado em sua composição (Vasconcellos et al., 1982; Kitchen et al., 1990; Schlindwein \& Anghinoni, 2000). Os altos teores no solo e o tempo de adoção do plantio direto podem ter causado essa ausência de diferença, especialmente se for considerado o nível de interpretação, que é alto nas duas formas de coleta. Em área por muito tempo sob plantio direto podem ocorrer, aleatoriamente, porções de solo com elevados teores de $\mathrm{P}$ em função do efeito residual de adubações anteriores. Stecker \& Brown (2001) observaram efeito residual de até sete anos para uma dose de $10 \mathrm{~kg} \mathrm{ha}^{-1}$ de $\mathrm{P}$ no sulco, com teores maiores que duas vezes em relação ao solo adjacente não adubado. Os mesmos autores estimaram em $6 \%$ a área superficial sob influência das zonas residuais de adubo. Como nesse estudo foram aplicados $18 \mathrm{~kg} \mathrm{ha}^{-1}$ de P na soja e $26 \mathrm{~kg} \mathrm{ha}^{-1}$ de $\mathrm{P}$ no feijão e no milho, é possível que o efeito residual dessas doses tenha influenciado no resultado das análises, pois a amostragem na entrelinha foi aleatória, sem identificação das linhas de semeadura dos cultivos anteriores ao feijão. Devese considerar também a dificuldade de centralizar a tradagem na linha de deposição do adubo, como observado por Kitchen et al. (1990) e Stecker \& Brown (2001), que pode diluir o teor de P na amostra que inclui a linha de adubação, e a profundidade de coleta, pois as maiores variações nos teores desse nutriente ocorrem nos $5 \mathrm{~cm}$ superficiais, conforme citado anteriormente, efeito que pode ter sido diluído com as coletas nas profundidades de $0-10$ e $0-20 \mathrm{~cm}$.

Apesar de o $\mathrm{P}$ e o $\mathrm{K}$ apresentarem elevada variabilidade horizontal no plantio direto (Souza et al., 1998; Silveira et al., 2000; Schlindwein \& Anghinoni, 2000; Alvarez V. \& Guarçoni, 2003), isso pode não interferir no resultado final médio da amostra quando a área está sob esse sistema há muito tempo. O mesmo ocorre com variações nos resultados dos demais atributos avaliados, não havendo mudança na interpretação agronômica do resultado da análise para recomendação de calagem e adubação.

\section{AGRADECIMENTOS}

À Fundação ABC (www.fundacaoabc.org.br) pelo apoio e disponibilização da área experimental.

\section{CONCLUSÕES}

1. Os teores dos atributos químicos $\mathrm{P}, \mathrm{K}, \mathrm{Ca}, \mathrm{Mg}$, carbono orgânico, $\mathrm{pH}$ e V foram maiores nas camadas mais superficiais do solo.

2. A distribuição de $\mathrm{K}$ em profundidade no solo não foi alterada pelas estratégias de adubação.

3. O teor de $\mathrm{P}$ na profundidade de $0-5 \mathrm{~cm}$ diminuiu com o uso contínuo (por mais de três anos) do sulcador tipo haste e com a ausência de adubação.

4. Os maiores teores dos atributos químicos avaliados no solo foram obtidos com a coleta da amostra de solo na profundidade de $0-10 \mathrm{~cm}$ em relação à coleta de $0-20 \mathrm{~cm}$.

5. Os teores de K, Ca e CTC foram maiores quando o método de coleta da amostra de solo incluiu a linha de semeadura, em relação à não inclusão da linha de semeadura.

6. Os métodos de coleta de amostras de solo com e sem inclusão da linha de semeadura, e as profundidades de coleta $0-10$ e $0-20 \mathrm{~cm}$ foram semelhantes quanto à interpretação dos resultados das análises para recomendação de calagem e adubação. 


\section{LITERATURA CITADA}

ALVAREZ V.,V.H. \& GUARÇONI, M.A. Variabilidade horizontal da fertilidade do solo de uma unidade de amostragem em sistema plantio direto. R. Bras. Ci. Solo, 27:297-310, 2003.

BORDOLI, J.M. \& MALLARINO, A.P. Deep and shallow banding of phosphorus and potassium as alternatives to broadcast fertilization for no-till corn. Agron. J., 90:2733, 1998.

COELHO, J.L.D. Avaliação de elementos sulcadores para semeadoras-adubadoras utilizadas em sistemas conservacionistas de manejo do solo. Campinas, Universidade Estadual de Campinas, 1998. 71p. (Tese de Mestrado).

COMISSÃO DE QUÍMICA E FERTILIDADE DO SOLO CQFRS/SC. Manual de adubação e calagem para os Estados do Rio Grande do Sul e Santa Catarina. 10.ed. Porto Alegre, Sociedade Brasileira de Ciência do Solo, 2004. 400p.

COMISSÃO DE FERTILIDADE DO SOLO DE MINAS GERAIS - CFSEMG. Recomendações para o uso de corretivos e fertilizantes em Minas Gerais. (5 ${ }^{\mathrm{a}}$ Aproximação). Viçosa, MG, 1999. 359p.

ECKERT, D.J. \& JOHNSON, J.W. Phosphorus fertilization in no-tillage corn production. Agron. J., 77:789-792, 1985.

ELTZ, F.L.F.; PEIXOTO, R.T.J. \& JASTER, F. Efeitos de sistemas de preparo do solo nas propriedades físicas de um Latossolo Bruno álico. R. Bras. Ci. Solo, 13:259-267, 1989.

EMPRESA BRASILEIRA DE PESQUISA AGROPECUÁRIA EMBRAPA/Fundação ABC. Mapa do Levantamento semidetalhado de solos: Município de Castro. Castro, 2001.

HOLANDA, F.S.R.; MENGEL, D.B.; PAULA, M.B.; CARVALHO, J.G. \& BERTONI, J.C. Influence of crop rotations and tillage systems on phosphorus and potassium stratification and root distribution in the soil profile. Comm. Soil Sci. Plant Anal., 29:2383-2394, 1998.

HOWARD, D.D.; ESSINGTON, M.E. \& LOGAN, J. Long-term broadcast and banded phosphorus fertilization of corn produced using two tillage systems. Agron. J., 94:51-56, 2002.

INSTITUTO AGRONOMICO DO PARANÁ - IAPAR. Cartas climáticas do Estado do Paraná, 2000. Disponível em: $<$ http://www.iapar.br/Sma/Cartas_Climaticas $>$. Acesso em jan., 2005.

KITCHEN, N.R.; HAVLIN, J.L. \& WESTFALL, D.G. Soil sampling under no-till banded phosphorus. Soil Sci. Soc. Am. J., 54:1661-1665, 1990.

KLEPKER, D. \& ANGHINONI, I. Características físicas e químicas do solo afetadas pro métodos de preparo e modos de adubação. R. Bras. Ci. Solo, 19:395-401, 1995.
MERTEN, G.H. \& MIELNICZUK, J. Distribuição do sistema radicular e dos nutrientes em Latossolo Roxo sob dois sistemas de preparo de solo. R. Bras. Ci. Solo, 15:369-374, 1991.

MONTGOMERY, D.C. Design and analysis of experiments. 5.ed. New York, John Wiley \& Sons, 2001. 684p.

MUZILLI, O. Influência do sistema de plantio direto, comparado ao convencional, sobre a fertilidade da camada arável do solo. R. Bras. Ci. Solo, 7:95-102, 1983.

NICOLODI, M.; ANGHINONI, I. \& SALET, R.L. Alternativa à coleta de uma secção transversal, com pá-de-corte, na largura da entrelinha, na amostragem do solo em lavouras com adubação em linha no sistema plantio direto. R. Plantio Direto, 69:22-28, 2002.

PAULETTI, V. Nutrientes: Teores e interpretações. Campinas, Fundação Cargill, 1998. 59p.

PAULETTI, V.; LIMA, M.R.; BARCIK, C. \& BITTENCOURT, A. Evolução nos atributos químicos de um Latossolo Vermelho sob diferentes métodos de preparo do solo. Sci. Agr., 6:9-14, 2005.

R DEVELOPMENT CORE TEAM. R: A language and environment for statistical computing. R Foundation for Statistical Computing. Vienna, 2005.<URL http://www.Rproject.org.>

RAIJ, B.van; CANTARELLA, H.; QUAGGIO, J.A. \& FURLANI, A.M.C., eds. Recomendação de calagem e adubação para o Estado de São Paulo. 2.ed. Campinas, Instituto Agronômico de Campinas, 1996. 285p. (Boletim Técnico, 100).

RAIJ, B.van; QUAGGIO, J.A.; CANTARELLA, H.; FERREIRA, M.E.; LOPES, A.S. \& BATAGGLIA, O.C. Análise química do solo para fins de fertilidade. Campinas, Fundação Cargill, 1987. 170p.

SEMEATO. Disponível em: <http://www.semeato.com.br $>$. Acesso em fev. de 2006

SCHLINDWEIN, J.A. \& ANGHINONI, I. Variabilidade horizontal de atributos de fertilidade e amostragem do solo no sistema plantio direto. R. Bras. Ci. Solo, 24:85-91, 2000 .

SILVEIRA, P.M.; ZIMMERMANN, F.J.P.; SILVA, S.C. \& CUNHA, A.A. Amostragem e variabilidade espacial de características químicas de um Latossolo submetido a diferentes sistemas de preparo. Pesq. Agropec. Bras., $35: 2057-2064,2000$.

SOUZA, L.S.; COGO, N.P. \& VIEIRA, S.R. Variabilidade de fósforo, potássio e matéria orgânica no solo em relação a sistemas de manejo. R. Bras. Ci. Solo, 22:77-86, 1998.

STECKER, J. \& BROWN, J.R. Soil phosphorus distribution and concentration from repeated starter phosphorus band application. Comm. Soil Sci. Plant Anal., 32:803-819, 2001. 
TISDALE, S.L.; NELSON, W.L. \& BEATON, J.D. Soil fertility and fertilizers. New York, Macmillan Publishing Company, 1985. 754p.
VASCONSELLOS, C.A.; SANTOS, H.L.; BAHIA FILHO, A.F.C.; OLIVEIRA, A.C. \& PACHECO, E.B. Amostragem de solo em área com adubação fosfatada aplicada a lanço e no sulco de plantio. R. Bras. Ci. Solo, 6:221-225, 1982. 